\title{
Robust Stability and Performance Analysis of Systems with Hysteresis Nonlinearities
}

\author{
Thomas E. Paré ${ }^{1}$ \\ Dept. of Mechanical Engineering \\ Email: tpareqsun-valley.stanford.edu
}

Jonathan P. How

Stanford University, Stanford CA 94305

\begin{abstract}
There has been extensive work done in recent years on the analysis and synthesis of systems having memoryless, sector bounded nonlinearities and uncertainties. In this paper we take a fundamentally different approach to develop tests of the stability of systems with hysteresis nonlinearities which, in general, have menory and are not sector bounded. Using an operator perspective, and considering a hysteresis that obeys a strict circulation direction, we develop a transformation which converts a hysteresis nonlinearity into a passive opcrator. Our main stability theorem then provides a simple Nyquist test (for a SISO system) or a linear matrix inequality (LMI) which is extended to include a provision for a robust performance test. A simple numerical example illustrates the benefit of the multiplier introduced for this class of nonlinearities.
\end{abstract}

\section{Introduction}

Hysteresis is a property of a wide range of physical systems and devices, such as electro-magnetic fields, mechanical ball bearings, and electronic relay circuits. The term hysteresis typically refers to the input-output relation between two time-dependent quantities that can not be expressed as a single-valued function. Instead, the relationship usually takes the form of loops that are traversed either in a clockwise or counter-clockwise direction. A hysteresis with counter-clockwise loops is referred to as a passive hysteresis [6, pg 366]. An example of a passive hysteresis is shown in Figure (1). This hysteresis could represent the relationship between the electric and magnetic fields of an electro-magnetic actuator. The area enclosed by the loops is often thought of as representing energy loss into the hysteretic element (see [8, pg 44], for example). Because this phenomenon is so prevalent, it is important to be able to predict its effect on systems in which it occurs. Early stability formulations for linear systems with hysteretic nonlinearities were done by Yakubovich $[12,1]$, who used a Lyapunov approach to arrive at a Popov-like test for stability, involving a multiplier of the form $(\tau+s) / s$. More recently, it was suggested that this multiplier be used for stability analysis of systems with hysteresis in an Integral Quadratic Constraint setting [7], although a result using this multiplier in an IQC was not presented. Other recent work [5] considered the time-derivative of the output of the nonlinear element as the basis for guaranteed stability of

\footnotetext{
${ }^{1}$ Corresponding author. Tel: (650) 723-3287; Fax: (650) 725-3377.
} $0-7803-4530-4 / 98 \$ 10.00$ (C) 1998 AACC

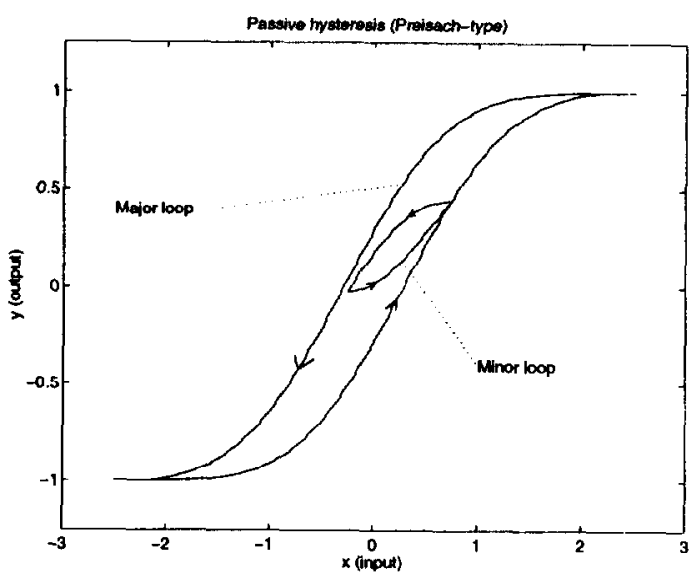

Fig. 1: Typical passive hysteresis characteristic.

systems with a Preisach hysteresis.

Because hysteresis is not memoryless and is typically not sector bounded, much of the work done in recent years in robust analysis, with the exception of work cited, is not applicable to systems with hysteresis nonlinearities. This paper addresses this issue. Our approach differs from previous work by taking a distinct operator perspective. In particular we show that, under the proper transformation, the passive hysteresis becomes a passive operator. This transformation establishes a mathematical connection between the passive hysteresis as an energy absorbing element and the property of a passive operator as having bounded extractable energy. We formulate this result using the basic integral properties of the hysteresis (i.e., counterclockwise circulation, etc.). This transformation then allows us to cast the problem into a passivity framework where we can then guarantee the $\mathcal{L}_{2}$-stability of systems containing these nonlinearities. Our main stability theorem leads to a simple graphical test in the Nyquist planc (for the SISO case) or to a particular linear matrix inequality (LMI) which can be readily extended to systems with multiple hystereses. We then extend the result to include a test for robust performance by using dissipation theory [11]. This again is expressed as an LMI, which is readily solved using existing optimization codes. Lastly, we present an example which illustrates the utility of the stability theorem.

The paper is organized as follows. Section 2 defines the notation used throughout the paper. Section 3 gives the properties of the passive hysteresis used to define a transformation which will make the hysteresis a passive operator. 
Section 4 presents a transformation and the Passivity Theorem to develop the robust stability tests. A simple numerical example is used in section 5 to illustrate the benefits of the analysis herein.

\section{Notation and Definitions}

For definitions of the vector space $\mathcal{L}_{2 e}, \mathcal{L}_{2 e}$-stability, $\mathcal{L}_{2^{-}}$ gain, or other terminology used in this paper, see, for example [4], or any equivalent text. Hysteresis (functional mapping with memory) $\Phi: \mathcal{L}_{2 e} \rightarrow \mathcal{L}_{2 e}$ such that for $\Phi: x \rightarrow y$ we have

$$
y(t)=\phi\left(x([0, t]), y_{0}\right) .
$$

For simplicity we will often write $y(t)=(\Phi x)(t)$.

Passive hysteresis $\Phi_{P}: \mathcal{L}_{2 e} \rightarrow \mathcal{L}_{2 e}$, having the input/output property with loops that are circuited counterclockwise. As a result, for bounded input $x\left(\left[t_{1}, t_{2}\right]\right)$, for some $A \geq 0$,

$$
-\int_{x\left(t_{1}\right)}^{x\left(t_{2}\right)} y d x \geq-A
$$

Similarly, an active hysteresis, $\Phi_{A}$ has loops that are strictly clockwise, and thus will have path integral with the lower bound

$$
\int_{x\left(t_{1}\right)}^{x\left(t_{2}\right)} y d x \geq-A .
$$

Passive operator $[4, \operatorname{pg} 173]$ is such that $\Phi: x \rightarrow y$, for some $\beta \geq 0$, satisfies

$$
\langle x, y\rangle_{T} \geq-\beta, \forall T \geq 0
$$

\section{Hysteresis Nonlinearity as a Passive Operator}

In order to use passivity theory to analyze systems with hysteresis, we need to establish a connection between a passive hysteresis as an element that absorbs energy over the long term, and a passive operator, which has a limited amount of extractable energy. This section provides such a connection using basic integral properties of a passive hysteresis. In particular, we define a transformation in Section 3.2 that converts a passive hysteresis into a passive operator using the basic integral properties of the nonlinearity. In Section 3.3 we show how a common passive hysteresis is readily converted into a passive operator under the transformation defined.

\subsection{Hysteresis Properties}

The class of passive hysteresis under consideration are characterized here by five properties that capture the basic integral properties of this nonlinearity.

1. Circulation is counter-clockwise. For any closed-path cycle, that is $\left(x\left(t_{1}\right), y\left(t_{1}\right)\right)=\left(x\left(t_{2}\right), y\left(t_{2}\right)\right)$ for some $t_{1} \leq t_{2}$, we have the path integral constraint:

$$
\oint_{x\left(t_{1}\right)}^{x\left(t_{2}\right)} y d x \leq 0
$$

For any closed path it follows that

$$
\oint_{y\left(t_{1}\right)}^{y\left(t_{2}\right)} x d y \geq 0
$$

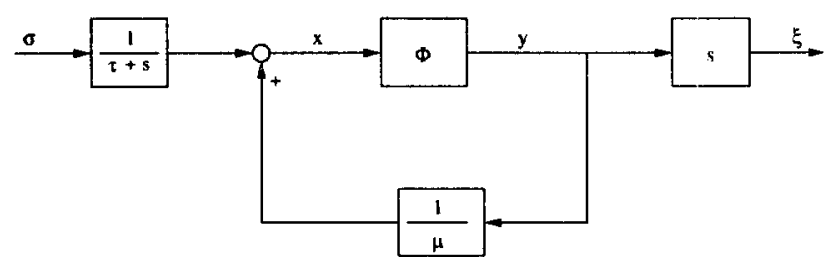

Fig. 2: Block diagram defining passive operator $\tilde{\Phi}: \sigma \rightarrow \xi$

2. The path integral (enclosed area) is lower bounded $[6$, $p g$ 365]. Let $\Gamma$ denote the path joining any two points $a$ and $b$ on the graph of the hysteresis, and note $\Gamma$ may involve many complete cycles (as in property 1). Let $\Gamma_{a b}$ denote the shortest path joining the two points $a$ and $b$, not containing any complete cycle, and let $p$ be any other point on the graph of the hysteresis.

Then we have that

$$
-\int_{\Gamma} y d x \geq-\int_{\Gamma_{u b}} y d x=\int_{\Gamma_{p}, \boldsymbol{t}} y d x-\int_{\Gamma_{p \rightarrow b}} y d x
$$

or, equivalently, using (4),

$$
\int_{\Gamma} x d y \geq \int_{\Gamma_{a b}} x d y=-\int_{\Gamma_{p \rightarrow a}} x d y+\int_{\Gamma_{p \rightarrow b}} x d y
$$

3. Area lower bound is independent of final point. There exists a point $p$ on the graph of hysteresis function such that,

$$
\int_{\Gamma_{p \rightarrow b}} x d y \geq 0
$$

which gives, using (5)

$$
-\int_{\Gamma_{p \rightarrow a}} x d y+\int_{\Gamma_{p \rightarrow b}} x d y \geq-\int_{\Gamma_{p \rightarrow a}} x d y
$$

4. Time derivative of input-output pairs are sector bounded. Let $\mu_{0}$ and $\mu_{1}$ be the minimum and maximum slopes on the hysteresis characteristic such that $\mu_{0} \leq y^{\prime}(x) \leq \mu_{1}$, where $y^{\prime}(x)$ is the local slope of the characteristic at $x$. As is typically done under the assumption of a loop transformation will assume that $\mu_{0}=0[10,12]$. This leads to the constraint

$$
\dot{x} \dot{y}-\frac{1}{\mu_{1}} \dot{y}^{2} \geq 0 .
$$

\subsection{Converting a Passive Hysteresis into a Passive Operator}

Here we will define a transformation which converts a passive hysteresis into a passive operator. The transformation that accomplishes this is given by a block diagram in Figure (2). The transformation will lead to a stability multiplier of the same form as that given in [1].

Definition 3.1 Given a hysteresis $\Phi_{P}: \mathcal{L}_{2 e} \rightarrow \mathcal{L}_{2 e}$, we define a new operator with characteristic given by the inputoutput relation $\tilde{\Phi}: \sigma \rightarrow \xi$ defined in Figure (2), with $\tau \geq 0$. 


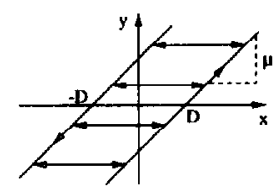

a: Backlash nonlinearity characteristic

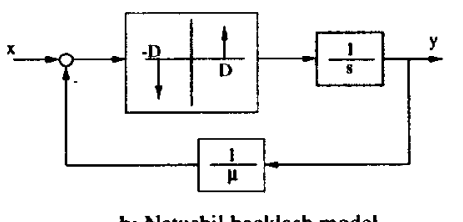

b: Netushil backlash model

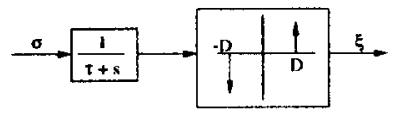

c: Tranformed backlash model

Fig. 3: (a.-b.) Backlash models.; (c.) Passive operator

Lemma 3.1 If $\Phi_{P}: \mathcal{L}_{2 e} \rightarrow \mathcal{L}_{2 e}$ is a passive hysteresis with properties (4)-( $(7)$, then $\tilde{\Phi}: \sigma \rightarrow \xi$ as given by Definition 3.1 is a passive operator.

Proof: By definition of $\tilde{\Phi}$ we have

$$
\begin{aligned}
\int_{0}^{T} \sigma \xi d t & =\int_{0}^{T}\left[\dot{x}-\frac{1}{\mu} \dot{y}+\tau\left(x-\frac{1}{\mu} y\right)\right] \dot{y} d t \\
& \geq \tau \int_{0}^{T}\left(x-\frac{1}{\mu} y\right) \dot{y} d t=\int_{y(0)}^{y(T)}\left(x-\frac{1}{\mu} y\right) d y \\
& \geq-\beta .
\end{aligned}
$$

Therefore, $\tilde{\Phi}$ is a passive operator.

The first inequality is a result of property (7), which restricts the derivative terms to be non-negative. This slope restriction, in The final step can be shown using the properties (4)-(6) as applied to a passive hysteresis that has an upper bound $\mu$ on the slope. Integration of the line integral as in (6) ultimately results in a lower bound on the path integral.

Note: The definition of a passive operator typically used has $\beta=0$ (see [5], [10, pg 352], for examples), since it is most commonly used in conjunction with sector-bounded nonlinearities. Here we require the more general definition $(\beta \geq 0)$ essentially bccause we are dealing with nonlinearities that have memory and are not sector bounded. In this case we require the relaxed lower bound in order to allow for an initial stored energy in the hysteresis.

\subsection{Example: The Backlash Nonlinearity}

Backlash, shown in Figure (3a) is an example of a hysteresis which can be transformed into a passive operator using Definition 3.1. Using the mathematical representation $[9$, pg 475] shown in Figure (3b), it is readily seen that under the transformation, the backlash involves a memoryless operator that is sector bounded $[0, \infty]$ (as depicted in Figure (3c). Then, having the $\frac{1}{\tau+s}$ multiplier at the input to the sector bounded nonlinearity maintains the passivity of the input-output relation, $\tilde{\Phi}: \sigma \rightarrow \xi$.

\section{Analysis of Systems with Hysteresis}

Using the transformation, Definition 3.1, we can now use passivity arguments to analyze the stability of systems containing passive hystereses. Here using standard passivity

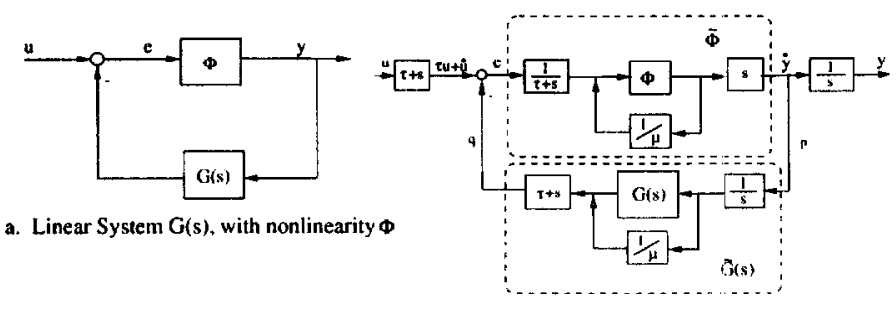

b. Transformed System $\tilde{G}($ s), with plassive operator, $\mathbb{\Phi}$

Fig. 4: (a.) Original system. (b.) Transformed system

techniques, we will use a loop transformation [10] to incorporate the transformation and then derive constraints on the linear portion of the system to guarantee robust stability. These constraints will be in the form of a linear matrix inequality (LMI), which is then extended to include bounds on a separate performance channel.

\subsection{Loop Transformation}

We assume that the total system to be analyzed has a nonlinearity, $\Phi$, that appears in a feedback configuration with a linear system $G(s)$, as depicted in Figure (4a). Introducing the transformation, as shown in Figure (4b), results in the system with modified feedforward and feedback elements, $\tilde{\Phi}$ and $\tilde{G}(s)$. Of course, if the original $\Phi$ was a passive hysteresis with the properties (4)-(7) given above, then $\tilde{\Phi}$ is a passive operator, according to Lemma 3.1. An essential feature of the loop transformation in Figure (4b) is that the input-output property of the net system from $u$ to $y$ is unchanged. Therefore, any stability conclusions we make regarding the transformed system will be applicable to the original system. We note here that if the original linear system $G$ has a state space representation

$$
G(s) \stackrel{s}{=}\left[\begin{array}{l|l}
A & B \\
\hline C & D
\end{array}\right]
$$

then a representation for the transformed system $\tilde{G}$ is

$$
\tilde{G}(s) \stackrel{s}{=}\left[\begin{array}{c|c}
\tilde{A} & \tilde{B}_{p} \\
\hline \tilde{C}_{q 1}+\tau \tilde{C}_{q 2} & \tilde{D}_{q p}
\end{array}\right],
$$

where

$$
\begin{array}{cc}
\tilde{A}=\left[\begin{array}{ll}
A & 0 \\
C & 0
\end{array}\right] & \tilde{B}_{p}=\left[\begin{array}{c}
B \\
D+1 / \mu
\end{array}\right] \\
\tilde{C}_{q 1}=\left[\begin{array}{ll}
C & 0
\end{array}\right] & \tilde{D}_{q p}=[D+1 / \mu] . \\
\tilde{C}_{q 2}=\left[\begin{array}{ll}
0 & 1
\end{array}\right] &
\end{array}
$$

\subsection{Robust Stability}

Here we use a form of the Passivity Theorem to derive the positive-real constraint on the linear portion of the system to guarantee the $\mathcal{L}_{2}$-stability of the closed loop system.

Theorem 4.1 The feedback relation $R_{f b}: u \rightarrow \dot{y}$ shown in Figure (4) is $\mathcal{L}_{2}$-stable if, for some $\delta>0$, we have the three conditions:

1. The fecdback element $\Phi$ is a passive hysteresis with properties (4)-(7), 
2. The linear system $\tilde{G}: p \rightarrow q$ is dissipative with respect to the supply rate:

$$
r(p, q)=p^{T} q-\delta p^{T} p
$$

3. $\dot{u} \in \mathcal{L}_{2}$.

As a result, we will have that $y(t) \rightarrow y_{s s}$, where the steady state value $\left|y_{s s}\right|<\infty$

Proof: First, note that by condition (3) we have that if $u \in \mathcal{L}_{2}$, then $\bar{u} \in \mathcal{L}_{2}$, where $\bar{u}=\tau u+\dot{u}$. Then by condition (1) we have that if $\Phi$ is a passive hysteresis, then by Lemma $3.1, \tilde{\Phi}$, (per Definition 3.1) is passive; i.e.,

$$
\langle e, \tilde{\Phi} e\rangle_{T} \geq-\beta,
$$

for some $\beta>0$, where, from Figure (4) $e=\bar{u}-q$. Next, Let $V(x)$ be a storage function [11] for the system $\tilde{G}$. Then by condition (2) we have that, with reference to Figure (4b),

$$
\begin{aligned}
\frac{d V}{d t} & \leq p^{T}(\bar{u}-e)-\delta p^{T} p \\
\Rightarrow \delta\left\|p_{T}\right\|_{2}^{2}-\left\|\bar{u}_{T}\right\|_{2}\left\|p_{T}\right\|_{2} & \leq V(x(0))+\beta .
\end{aligned}
$$

Completing the square and simplifying yields

$$
\left\|p_{T}\right\|_{2} \leq \frac{1}{\delta}\left[\|\bar{u}\|_{2}+(\delta(V(x(0))+\beta))^{1 / 2}\right],
$$

and, hence, the feedback relation $R_{f b}: \bar{u} \rightarrow p$ is $\mathcal{L}_{2}$-stable. It follows then since $p=\dot{y} \in \mathcal{L}_{2}$, as $t \rightarrow \infty$ we have $\dot{y} \rightarrow 0$. We conclude then that $y(t) \rightarrow y_{s s}$, where steady state $y_{s s}$ is bounded.

4.2.1 LMI Test for Robust Stability: Condition 2 of Theorem 4.1 can be expressed as LMI. By letting $x$ be the state of system $\tilde{G}: p \rightarrow q$ and the storage function $V(x)=\frac{1}{2} x^{T} P x$ where $P>0$, then we have

$$
p^{T} q-\delta p^{T} p-x^{T} P \dot{x}=\frac{1}{2}\left[\begin{array}{l}
x \\
p
\end{array}\right]^{T}\left[\begin{array}{ll}
N_{11} & N_{12} \\
N_{12}^{T} & N_{22}
\end{array}\right]\left[\begin{array}{l}
x \\
p
\end{array}\right] .
$$

where $N_{11}=-\tilde{A}^{T} P-P \tilde{A}, N_{12}=\left(\tilde{C}_{q 1}+\tau \tilde{C}_{q 2}\right)^{T}-P \tilde{B}_{p}$ and $N_{22}=\tilde{D}_{q p}+\tilde{D}_{q p}^{T}-2 \delta I$. Assuming that conditions 1 and 3 of hold, then an equivalent test of stability for the closed loop system becomes

$$
\begin{aligned}
& \delta>0, \tau>0, P>0 \\
& {\left[\begin{array}{ll}
N_{11} & N_{12} \\
N_{12}^{T} & N_{22}
\end{array}\right] \geq 0}
\end{aligned}
$$

4.2.2 Graphical Test for Stability: An equivalent condition for the strict passivity of an $L T I$ sytem, $H(s)$ is that $H(j \omega)+H^{*}(j \omega)>0, \forall \omega \in \mathcal{R}([10, \mathrm{pg} 223])$, which is equivalent to $H(s)$ being strictly positive real. For the SISO system $\tilde{G}_{q p}=\frac{\tau+s}{s}(G(s)+1 / \mu)$, where the original system $G \in R H_{\infty}$, it is straightforward to show that we can test for the existence of a $\tau \geq 0$ that will satisfy the strict passivity of $\tilde{G}_{q p}$ using the Nyquist plot of the original system. In particular, we have that $\exists \tau \geq 0$ for which $\tilde{G}_{q p}$ is positive real if the graph of $G(j \omega), \forall \omega \geq 0$ does not enter the portion of third quadrant of the Nyquist plane to the left of the point $(-1 / \mu, 0)$. This graphical test is depicted in Figure (5).

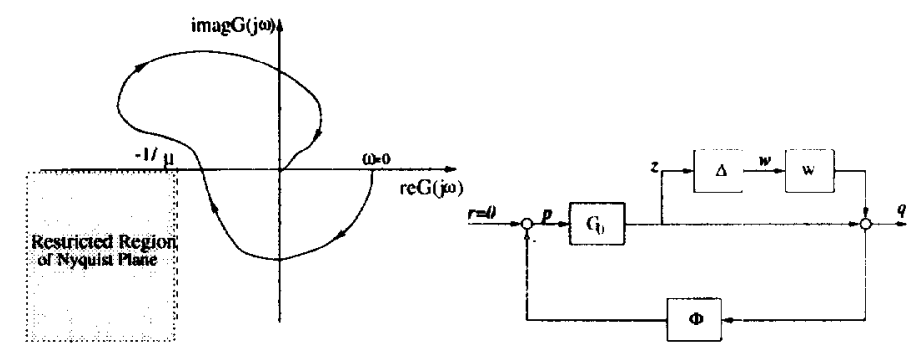

Fig. 5: Nyquist test.

Fig. 6: Example system.

\subsection{Robust Performance}

We can extend the analysis techniques for robust stability to include a norm bounded constraint, that may correspond to a desired level of performance or to some level of uncertainty. For example, consider the nonlinear feedback system with the channel from $w$ to $z$, as shown in Figure (6), representing multiplicative uncertainty [13] in the output of $G_{0}$. The $\Delta$-block in Figure (6) could represent an additional uncertainty in the system or an $\mathcal{H}_{\infty}$ performance metric. We can develop the robust performance analysis by simply augmenting the supply rate (10) with a term corresponding to the system performance (see [2, pg 124] for a similar example). With the supply rate

$$
r(p, q, w, z)=\gamma^{2} w^{T} w-z^{T} z+\lambda p^{T} q-\delta p^{T} p,
$$

we can then bound the $\mathcal{L}_{2}$ performance by solving the optimization problem,

$$
\begin{aligned}
\text { minimize } & \gamma^{2} \\
\text { subject to: } & \lambda, \tau, \delta>0, M \geq 0, P>0
\end{aligned}
$$

where

$$
M=\left[\begin{array}{lll}
M_{11} & M_{12} & M_{13} \\
M_{12}^{T} & M_{22} & M_{13} \\
M_{13}^{T} & M_{23}^{T} & M_{33}
\end{array}\right]
$$

with $M_{11}=-\tilde{A}^{T} P-P \tilde{A}-\tilde{C}_{z}^{T} \tilde{C}_{z}, M_{12}=\lambda \tilde{C}_{q 1}^{T}+\tau \tilde{C}_{q 2}^{T}-$ $P \tilde{B}_{p}-\tilde{C}_{z}^{T} \tilde{D}_{z p}, M_{13}=-P \tilde{B}_{w}-\tilde{C}_{z}^{T} \tilde{D}_{z w}, M_{22}=\lambda\left(\tilde{D}_{q p}+\right.$ $\left.\tilde{D}_{q p}^{T}\right)-2 \delta I-\tilde{D}_{z p}^{T} \tilde{D}_{z p}, M_{23}=\lambda \tilde{D}_{q w}-\tilde{D}_{z p}^{T} \tilde{D}_{z w}$, and $M_{33}=$ $\gamma^{2}-\tilde{D}_{z w}^{T} \tilde{D}_{z w}$.

\section{Numerical Example}

Here we will use the analysis given above to test the robust stability of a system with a passive hysteresis and plant output multiplicative uncertainty. Consider the nominal system, $G_{0}(s)$ shown in Figure (6), with passive hysteresis, $\Phi$, in the feedback channel, and a norm bounded $\Delta$, which in this case corresponds to an additional plant uncertainty. Using standard practice we let $\Delta \in \Delta$ where $\Delta=\left\{\Delta \in R H_{\infty}:\|\Delta\| \leq 1\right\}$. Our approach is to minimize the upper bound on $\gamma$, which is the $\mathcal{L}_{2}-$ gain from $w$ to $z$. If this upper bound is less than unity, then we can conclude that the system will be stable $\forall \Delta \in \Delta$. We will then examine the conservativeness of the upper bound by considering particular $\Delta$ that exceed the norm bound. Consider $G_{0}(s)$ and the weighting function, $W(s)$, given by

$$
G_{0}(s)=7.5 \frac{s^{2}-0.2 s+0.1}{s^{3}+2 s^{2}+2 s+1}, \quad W(s)=0.97 \frac{s}{s+10},
$$



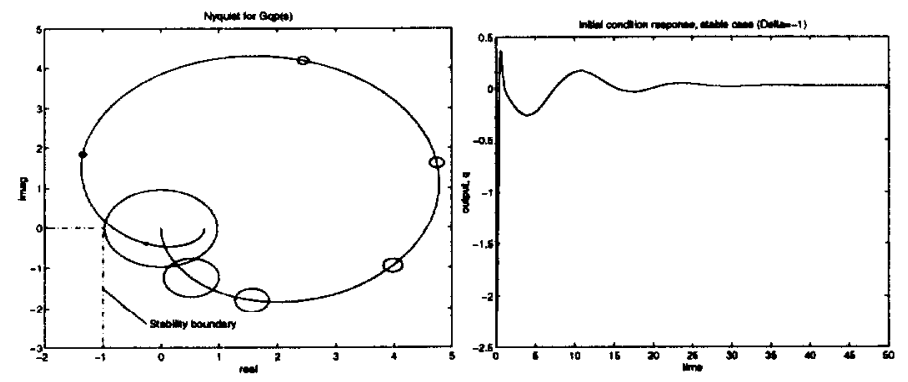

Fig. 7: Nyquist for $G_{0}(s)$. Fig. 8: I.C. resp. $\Delta=-1$
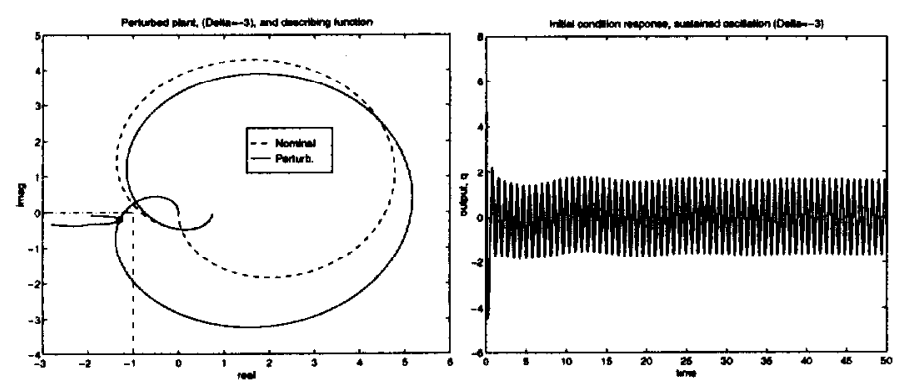

Fig. 9: Nyquist, $\Delta=-3$ Fig. 10: 1.C. resp. $\Delta=-3$

with the nonlinearity a Preisach type with $\mu=1$, as shown in Figure (1). Augmenting the system with the stability multiplier, using the system representation $(8,9)$, and solving the optimization problem (12) yields the minimized upper bound, $\gamma_{o p t}=0.99$, which indicates robust stability over the set of dynamic uncertainty $\Delta$. The corresponding stability (multiplier) parameters are $\tau=1.129, \lambda=2.097$, and $\delta=1.99 e^{-9}$. This stability condition is consistent with the Nyquist plot, and uncertainty ellipses, of the nominal plant $G_{0}$, shown in Figure (7), avoiding the restricted region of the lower left quadrant. A typical initial condition response for the case $\Delta=-1$, Figure (8), indicates that the system is robustly stable.

Note that without the stability multiplier, $(\tau=0)$, the stability test reduces to that considered in [7] and in this case the analysis would fail for this system with $\mu=1$. Thus, for this system we have established a less conservative test. However, this test is still conservative to a certain degree. Consider the stability when $\Delta \notin \Delta$. For this system the onset of instability occurs when the perturbation reaches $\Delta=-3$, as indicated by a sustained 1.3 $\mathrm{Hz}$ oscillation in Figure (10). At this perturbation level, the Nyquist plot extends well into the third quadrant, and intersects the describing function for the nonlinearity. The intersection of the Nyquist and describing function plots (see Figure (9)) does predict a sustained oscillation at this frequency, (see [3, pg 66]). In this case, comparing the describing function to the stability region outlined in Figure (9) gives a good indication of the conservativeness of the multiplier analysis. As the Nyquist plot indicates, the system at this level of perturbation is very active, and extends well into the left hand plane. As depicted by the limit cycle in Figure (10), the passive hysteresis is absorbing energy at a rate equal to the rate at which it is produced by the system.

\section{Conclusions}

In this paper we have investigated the stability of systems with hysteresis nonlinearities. By restricting our attention to hysteresis that has strictly counter-clockwise circulation we motivated the use a a particular transformation which converts this nonlinearity into a passive operator. The transformation is subsequently used in a passivity framework to develop a stability theorem for systems having nonlinearities with the prescribed characteristics. This stability test, for the SISO case, is easily verified by a simple graphical test in the Nyquist plane, and is readily computed by solving an LMI. The LMI framework allows for a straightforward extension of the test to include robust performance. A simple numerical example given illustrates the utility of this particular form of the multiplier in testing for the robust stability of a linear system with a hysteresis nonlinearity and a multiplicative uncertainty in the plant output.

\section{References}

[1] N. Barabanov and V. Yakubovich. Absolute Stability of Control Systems with One Hysteresis Nonlinearity. Avtomatika i Telemekhanika, 12:5-12, Dec. 1979.

[2] S. Boyd, L. E. Ghaoui, E. Feron, and V. Balakrishan. Linear Matrix Inequalities in System and Control Theory. SIAM, 1994.

[3] P. A. Cook. Nonlinear Dynamical Systems. PrenticeHall, 1994.

[4] C. A. Desoer and M. Vidyasagar. Feedback Systems: Input-Output Properties. Prentice-Hall, Englewood Cliffs, NJ, 1975.

[5] R. B. Gorbet, K. Morris, and D. Wang. Stability of control for the preisach hysteresis model. In IEEE Conf. on Robotics and Automation, volume 1, pages 241-247, 1997.

[6] J. C. Hsu and A. U. Meyer. Modern Control Principles and Applications. McGraw-Hill, 1968.

[7] U. Jönsson. Stability of uncertain systems with hysteresis nonlinearities. Technical report, Dept. of Automatic Control, Lund Inst. of Tech., 1996.

[8] I. D. Mayergoyz. Mathematical Models of Hysteresis. Springer-Verlag, New York, NY, 1991.

[9] A. Netushil. Theory of Automatic Control. Mir Publishers, 1973.

[10] M. Vidyasagar. Nonlinear Systems Analysis, 2nd Ed. Academic Press, New York, NY, 1993.

[11] J. C. Willems. Dissipative dynamical systems part II: Linear systems with quadratic supply rates. In Archive Rational Mechanics Analysis, volume 45. 1972.

[12] V. Yakubovich. The method of matrix inequalities in the stability theory of nonlinear nontrol systems, III. Absolute Stability of Systems with Hysteresis Nonlinearities. Automation and Remote Control, 26:753-763, Apr. 1967.

[13] K. Zhou. Essentials of Robust Control. Prentice-Hall, 1998. 